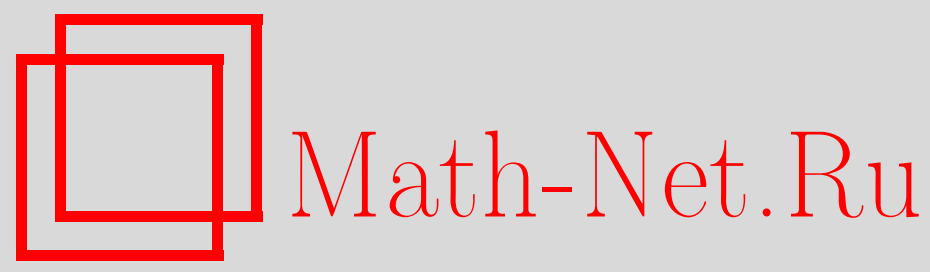

М. Б. Хрипунова, Аддитивные задачи с числами, имеющими заданное число простых делителей, Матем. заметки, 1998, том 63, выпуск 5, 749-762

DOI: https://doi.org/10.4213/mzm1342

Использование Общероссийского математического портала Math-Net.Ru подразумевает, что вы прочитали и согласны с пользовательским соглашением http://www . mathnet.ru/rus/agreement

Параметры загрузки:

IP : 54.198 .67 .100

26 апреля 2023 г., 15:30:50

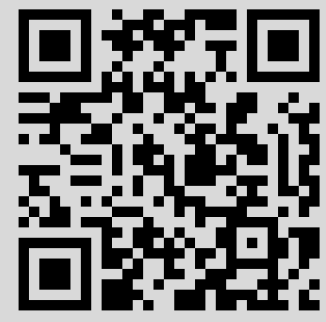




\section{АДДИТИВНЫЕ ЗАДАЧИ С ЧИСЛАМИ, ИМЕЮЩИМИ ЗАДАННОЕ ЧИСЛО ПРОСТЫХ ДЕЛИТЕЛЕЙ}

\section{М. Б. Хрипунова}

Изучается асимптотика сумм вида

$$
\sum_{\substack{n<N \\ \omega(n)=k}} \tau(|b n-a|)
$$

где $\omega(n)$ - число различных простых делителей числа $n$, а $\tau(n)$ - число всех его делителей.

Библиографоия: 19 названий.

Пусть $\omega(n)$ - число различных простых делителей $n$, а $\tau(n)$ - число делителей натурального числа $n$. Целью настоящей статьи является нахождение асимптотики суммы

$$
\sum_{\substack{n<N \\ \omega(n)=k}}^{\prime} \tau(|b n-a|)
$$

равномерной по $k, a, b$ при $k \leqslant \gamma \ln _{2} N,(a, b)=1,|a|<c N,|b| \leqslant(\ln N)^{\alpha} ; N$ - большое натуральное число, $\gamma, c, \alpha$ - фиксированные постоянные, $\sum^{\prime}$ означает, что в сумме (1) $b n-a \neq 0$. Здесь и далее $\ln _{2} N=\ln \ln N, \ln _{3} N=\ln \ln \ln N$.

При $a=N, b=1$, находя асимптотику суммы (1), мы тем самым находим асимптотику числа решений уравнения $N=n+x y$, где $\omega(n)=k, x, y$ - натуральные числа; а при $b=1$ - асимптотику числа решений уравнения $a=n-x y$, где $n<N, \omega(n)=k, x, y-$ натуральные числа.

Наиболее просто асимптотика выглядит в случае $b=1, a=1$.

Tеорема 1. При $k \leqslant \gamma \ln _{2} N$ имеем

$$
\sum_{\substack{n<N \\ \omega(n)=k}} \tau(n-1)=C \frac{N}{\Gamma(\sigma+1)} \frac{\left(\ln _{2} N\right)^{k-1}}{(k-1) !} \prod_{p}\left(1-\frac{1}{p}\right)^{\sigma}\left(1+\sigma \frac{\lambda(p)}{p-1}\right)\left(1+O\left(\frac{\left(\ln _{3} N\right)^{2}}{\ln _{2} N}\right)\right),
$$

$2 \partial e$

$$
C=\prod_{p}\left(1+\frac{1}{p(p-1)}\right), \quad \lambda(p)=1-\frac{p}{p^{2}-p+1}, \quad \sigma=\frac{k-1}{\ln _{2} N}
$$

Работа выполнена при финансовой поддержке Российского фонда фундаментальных исследований, грант № 96-01-00502. 
Данную задачу можно считать аналогом проблемы Титчмарша, которая заключается в нахождении асимптотики суммы $\sum_{p<N} \tau(p-1), p$ - простое число. Эта проблема была решена Титчмаршем [1] в 1930 году с помошью расширенной гипотезы Римана. Ее безусловное решение получил в 60-е годы Ю. В. Линник [2], используя созданньй им дисперсионный метод. В последующие годы обобщением проблемы Титчмарша занимались многие авторы: Б. М. Бредихин [3], М. Б. Барбан [4], А. К. Каршнев [5], Ж. А. Пиядина [6], А. Фуджи [7] и др. Они находили асимптотики сумм вида

$$
\sum_{n<N} \tau(n+a)
$$

где $n$ равно произведению фиксированного числа простых чисел, удовлетворяющих некоторым условиям. К примеру, у Пиядиной [6] $n$ имеет не более шести простых делителей больших $N^{1 / 883}$.

Настоящая работа является продолжением работ [8] и [9], в которых находятся асимптотики сумм

$$
\sum_{\substack{n<N \\ \Omega(n)=k}} \tau(n-1), \quad \sum_{\substack{n<N \\ \Omega(n)=k}} \tau(N-n)
$$

равномерно по $k \leqslant(2-\varepsilon) \ln _{2} N$ для обеих сумм и $k \in\left[(2+\varepsilon) \ln _{2} N, b \ln _{2} N\right]$ для второй суммы. Здесь и далее $\Omega(n)$ - число простых делителей $n$ с учетом их кратности.

Действуя по схеме работы [9], можно доказать следующую теорему.

Tеорема 2. Пусть $k \leqslant(2-\varepsilon) \ln _{2} N,(a, b)=1,|a|<c N,|b| \leqslant(\ln N)^{\alpha} ; c, \alpha-$ постоянные, $N$-большое натуральное число. Тогда

$$
\begin{aligned}
& \sum_{\substack{n<N \\
\Omega(n)=k}}^{\prime} \tau(|b n-a|)=\prod_{p}\left(1+\frac{1}{p(p-1)}\right) \frac{N\left(\ln _{2} N\right)^{k-1}}{(k-1) !} \\
& \quad \times \Phi(\sigma) E(a b, \sigma) E(\sigma)\left(1+O\left(\frac{\left(\ln _{3} N\right)^{3}}{\ln _{2} N}\right)\right),
\end{aligned}
$$

əде $\sigma=k / \ln _{2} N$,

$$
\begin{gathered}
\Phi(\sigma)=\frac{1}{\Gamma(\sigma+1)} \prod_{p}\left(1-\frac{1}{p}\right)^{\sigma}\left(1-\frac{\sigma}{p^{2}-p+1}\right)^{-1}, \quad E(\sigma)=\prod_{p}\left(1-\frac{\sigma}{p^{2}-p+1}\right) \\
E(a b, \sigma)=\prod_{p \mid a b}\left(1-\frac{\sigma}{p^{2}-p+1}\right)^{-1} \prod_{p \mid a}\left(1+\sum_{r<\alpha(a, p)} \frac{\sigma}{p^{r}}\right) \\
+\left(\frac{p^{2}-p+1}{(p-1)^{2}}-\frac{\sigma}{(p-1)^{2}}\right)\left(\frac{\sigma}{p}\right)^{\alpha(a, p)}
\end{gathered}
$$

$\alpha(a, p)$ - показатель степени, с которым р входит в каноническое разложение $а$. $\sum^{\prime}$ означает, что в этой сумме $b n-a \neq 0$.

Сформулируем основной результат данной работы. 
Tеорема 3. Пусть $k \leqslant \gamma \ln _{2} N,(a, b)=1,|a|<c N,|b| \leqslant(\ln N)^{\alpha}, N-$ большое натуральное число, $\gamma, c, \alpha-$ постоянные. Тогда

$$
\begin{aligned}
\sum_{n<N, \omega(n)=k}^{\prime} \tau(|b n-a|)= & C \frac{N}{\Gamma(\sigma+1)} \frac{\left(\ln _{2} N\right)^{k-1}}{(k-1) !} \prod_{p}\left(1-\frac{1}{p}\right)^{\sigma}\left(1+\sigma \frac{\lambda(p)}{p-1}\right) \\
& \times E(a, b, \sigma)\left(1+O\left(\frac{\left(\ln _{3} N\right)^{2}}{\ln _{2} N}\right)\right)
\end{aligned}
$$

$2 \partial e$

$$
\begin{array}{r}
C=\prod_{p}\left(1+\frac{1}{p(p+1)}\right), \quad \lambda(p)=1-\frac{p}{p^{2}-p+1}, \quad \sigma=\frac{k-1}{\ln _{2} N}, \\
E(a, b, \sigma)=\prod_{p \mid a}\left(\lambda(p)+\sigma \frac{\lambda(p)}{p-1}+\sigma\left(1-\frac{1}{p}\right)^{-2}\left(\frac{1}{p}-\frac{1}{p^{\alpha(a, p)}}\left(1+\frac{\lambda(p)}{p-1}\right)\right.\right. \\
\left.\left.\quad \times\left(1+\frac{\sigma}{p-1}\right)\right)\right) \prod_{p \mid b}\left(1+\frac{\sigma}{p-1}\right) \lambda(p) \prod_{p \mid a b}\left(1+\sigma \frac{\lambda(p)}{p-1}\right)^{-1},
\end{array}
$$

$\alpha(a, p)$ - наибольший показатель степени, в которой $p$ делит $a, \sum^{\prime}$ означает, что в этой сумме $b n-a \neq 0$.

При доказательстве этой теоремы в отличие от работ [8] и [9] приходится сталкиваться с дополнительньми трудностями, возникающими из-за различий в свойствах функций $\Omega(n)$ и $\omega(n)$. Несмотря на схожесть этих функций, $\Omega(n)$ вполне аддитивная, a $\omega(n)$ просто аддитивная. Это и вносит дополнительные сложности, которые нашли свое отражение при нахождении асимптотики сумм

$$
\sum_{\substack{n \leqslant x \\ \Omega(n)=k}} 1
$$

Асимптотика (2) при всех возможных $k, k \notin\left[(2-\varepsilon) \ln _{2} N,(2+\varepsilon) \ln _{2} N\right]$, была найдена в работах [10]-[12] довольно давно, а асимптотическоеповедение (3) при $k \leqslant \ln N\left(\ln _{2} N\right)^{-2}$ было получено сравнительно недавно в работе [13].

Далее приведем необходимые вспомогательные результаты.

Пусть $|A|$ - число элементов множества $A, P(t)=\prod_{p \leqslant t} p, p$ - простое число,

$$
\begin{gathered}
\pi(x, t, k)=|\{n: n \in[1, x],(n, P(t))=1, \omega(n)=k\}| \\
\pi(x, a, d, k)=\mid\{n: n \leqslant x], n \equiv a(\bmod d), \omega(n)=k\} \mid
\end{gathered}
$$

При нахождении асимптотики суммы (2) используем следующую лемму. 
Лемма $1[14$, лемма 5] (см. также [15, теорема 6]). Пусть $R>0$. Тогда равномерно no

имеем

$$
\frac{3}{2} \leqslant t<\exp \left((\ln x)^{2 / 5}\right), \quad x \geqslant 3, \quad 1 \leqslant k \leqslant R \ln U
$$

$$
\pi(x, t, k)=g(r, t) \frac{x}{\ln x} \frac{(\ln U)^{k-1}}{(k-1) !}\left(1+O_{R}\left(\frac{1}{\ln _{2} x}\right)\right),
$$

әде

$$
\begin{gathered}
r=\frac{k-1}{\ln U}, \quad U=\frac{\ln x}{\ln t}, \\
g(r, t)=\frac{1}{\Gamma(r+1)}\left((\ln t)^{r} \prod_{p \leqslant t}\left(1-\frac{1}{p}\right)^{r}\right) \prod_{p>t}\left(1-\frac{1}{p}\right)^{r}\left(1+\frac{r}{p-1}\right) .
\end{gathered}
$$

Далее, пусть

$$
\mu(x, k, t, a, d)=|\{n: n \leqslant x, \Omega(n)=k,(n, P(t))=1, n \equiv a(\bmod d)\}| .
$$

Следующая лемма, которую можно назвать аналогом теоремы Виноградова-Бомбьери, доказана в работе [8].

Лемма 2. Пусть $2 \leqslant t \leqslant \sqrt{x}, k \leqslant\left(\ln _{2} x\right)^{2} u$

$\Delta_{k}(t)=\sum_{d \leqslant Q} \max _{y \leqslant x} \max _{(a, d)=1}\left|\mu(y, k, t, a, d)-\frac{1}{\varphi(d)}\right|\{n: n \leqslant y, \Omega(n)=k,(n, d P(t))=1\}||$.

Тогда $\Delta_{k}(t) \ll Q \sqrt{x} \exp \left(\left(\ln _{2} x\right)^{2+\varepsilon}\right)+x(\ln x)^{-B}$, где $\varepsilon>0, B$ - любая положительная постоянная.

Лемма 3. При $d \leqslant x^{1-\alpha}, 0<\alpha<1 / 2, k \leqslant b \ln _{2} x$

$$
\max _{(a, k)=1} \pi(x, a, d, k) \ll \frac{x}{\varphi(d) \ln x} \frac{\left(\ln _{2} x\right)^{k}}{k !} \sqrt{k} .
$$

ДокАЗАТЕЛЬСтво. В работе [16] для неотрицательных мультипликативных функций, удовлетворяющих условиям $f\left(p^{r}\right) \leqslant A_{1}^{r}, r \geqslant 1, f(n) \leqslant A_{2} n^{\varepsilon}, \varepsilon>0$, доказано, что при $d<x^{1-\alpha}$

$$
\max _{(a, d)=1} \sum_{\substack{n \leqslant x \\ n \equiv a(\bmod d)}} f(n) \ll \frac{x}{\varphi(d)} \frac{1}{\ln x} \exp \left(\sum_{p \leqslant x} \frac{f(p)}{p}\right) .
$$

Отсюда при $1 \leqslant k \leqslant b \ln _{2} x$ получим

$$
\begin{aligned}
\pi(x, a, d, k) & \leqslant \sum_{\substack{n \leqslant x \\
n \equiv a(\bmod d)}}\left(\frac{k}{\ln _{2} x}\right)^{\omega(n)}\left(\frac{\ln _{2} x}{k}\right)^{k} \\
& \ll \frac{x}{\varphi(d) \ln x} \exp \left(\frac{k}{\ln 2 x} \sum_{p \leqslant x} \frac{1}{p}\right)\left(\frac{\ln _{2} x}{k}\right)^{k} \ll \frac{x}{\varphi(d) \ln x}\left(e \frac{\ln _{2} x}{k}\right)^{k} .
\end{aligned}
$$

Применяя формулу Стирлинга, получим

т.е. получим утверждение леммы.

$$
\left(\frac{\ln _{2} x}{k} e\right)^{k} \frac{k !}{\left(\ln _{2} x\right)^{k}} \ll \frac{e^{k}}{k^{k}} k^{k} e^{-k} \sqrt{k},
$$


Лемма 4. Пусть $N$ - натуральное число. Тогда

$$
\sum_{d \mid N} \frac{\ln d}{d} \gamma^{\omega(d)} \leqslant\left(\ln _{2} N\right)^{\gamma+1}, \quad \sum_{d \mid N} \frac{\gamma^{\omega(\delta)}}{\delta} \ll\left(\ln _{2} N\right)^{\gamma}
$$

Eсли $n_{1}-$ числа, все простье делители которых меньие либо равны $t$, то

$$
\sum_{n_{1} \leqslant y} \frac{\gamma^{\omega\left(n_{1}\right)}}{n_{1}} \ll(\ln t)^{\gamma}
$$

ДокАЗАтЕЛьство. Действительно,

$$
\sum_{\delta \mid N} \frac{\gamma^{\omega(\delta)}}{\delta} \ll \exp \left(\gamma \sum_{p \leqslant \ln N} \frac{1}{p}+\gamma \sum_{\substack{p \mid N \\ p>\ln N}} \frac{1}{p}\right) \ll\left(\ln _{2} N\right)^{\gamma}
$$

Далее, имеем

$$
\begin{gathered}
\sum_{d \mid N} \frac{\ln d}{d} \gamma^{\omega(d)} \leqslant \gamma \sum_{p^{r} \mid N} \frac{\ln p^{r}}{p^{r}} \sum_{d \mid N} \frac{\gamma^{\omega(d)}}{d} \ll\left(\sum_{p \leqslant(\ln N)^{2}} \frac{\ln p}{p}+O(1)\right) \ln _{2}^{\gamma} N \ll\left(\ln _{2} N\right)^{\gamma+1} \\
\sum_{n_{1} \leqslant y} \frac{\gamma^{\omega\left(n_{1}\right)}}{n_{1}} \leqslant \prod_{p \leqslant t}\left(1+\gamma \sum_{r=1}^{\infty} \frac{1}{p^{r}}\right) \ll \exp \left(\sum_{p \leqslant t} \frac{\gamma}{p}\right) \ll(\ln t)^{\gamma} .
\end{gathered}
$$

Лемма 5. Пусть а-натуральное число. Тогда

$\sum_{\substack{d<N \\(d, a)=1}} \frac{1}{\varphi(d)}=\prod_{p}\left(1+\frac{1}{p(p-1)}\right) \prod_{p \mid a}\left(1-\frac{1}{p}\right)\left(1+\frac{1}{p(p+1)}\right)^{-1} \ln N+O\left(\sum_{d \mid a} \frac{1+\ln d}{d}\right)$.

Доказательство леммы 5 можно найти во многих работах, например, [17, лемма 3.2].

ДоКАЗАТЕЛЬСТВО ТЕОРЕМЫ 3 . Представим $n$ в сумме (1) в виде $n_{1} n_{2}$, где у $n_{1}$ все простые делители не больше $t=\exp \left(\left(\ln _{2} N\right)^{2}\right)$, а у $n_{2}$ все простые делители больше $t$; $n_{1}$ и $n_{2}$ могут равняться 1 . Пусть

$$
U=\max \left\{N, \max _{n \leqslant N}|b n-a|\right\}
$$

$A$ - большая положительная постоянная. Заметим, что $b n \ll N(\ln N)^{\alpha}$, иньпи словами, $U \ll N(\ln N)^{\alpha}$.

Сначала оценим часть суммы (1), в которой $n_{1}>y, y=\exp \left(\left(\ln _{2} N\right)^{4}\right)$. Применив неравенство Коши, получим, что эта часть суммы

$$
\ll \sqrt{\sum_{n_{1}<y} \sum_{n_{2}<N / n_{1}} 1 \cdot \sum_{m<U} \tau^{2}(m)} .
$$


Далее, $\sum_{m<U} \tau^{2}(m) \ll U(\ln U)^{3}$ (см. [18]). Оценим сумму (1) по $n_{1}>y$ с помощью леммы 5.2 из [19]:

$$
\ll N \sum_{y<n_{1} \leqslant N} \frac{1}{n_{1}} \ll N \ln N \exp \left(-\frac{\ln _{3} t}{\ln t} \ln y+2 \ln _{2} t\right) .
$$

Учитьвая, что $\ln y / \ln t \geqslant\left(\ln _{2} N\right)^{2}$, выражение (4)

$$
\ll \sqrt{\frac{N}{(\ln N)^{2 A+3+\alpha}} U(\ln U)^{3}} \ll \frac{N}{\ln ^{A} N} .
$$

Кроме того, можно считать, что в сумме (1) $n$ и $a$ не имеют общих простых делителей больших $t$. Действительно, та часть суммы, в которой есть такие $n$, будет

$$
\begin{aligned}
& \leqslant \sum_{\substack{p^{r} \| a \\
p>t}} \tau\left(p^{r}\right) \sum_{n<N / p^{r}} \tau\left(\left|b n-\frac{a}{p^{r}}\right|\right) \leqslant \sum_{\substack{p^{r} \mid a \\
p>t}} \tau\left(p^{r}\right) \sum_{m \leqslant U / p^{r}} \tau(m) \\
& \ll U \ln U \sum_{\substack{p^{r} \mid a \\
p>t}} \frac{\tau\left(p^{r}\right)}{p^{r}} \ll U \ln ^{2} U \sum_{\substack{p \mid a \\
p>t}} \frac{1}{p} \ll \frac{U \ln ^{3} U}{t} \ll \frac{N}{\ln ^{A} N} .
\end{aligned}
$$

Далее можно считать, что $n$ не делится на $p^{r}$ при $r \geqslant 2, p>t$. Действительно, эта часть суммы будет

$$
\ll \sqrt{\sum_{p>t} \frac{N}{p^{2}} \cdot \sum_{m<U} \tau^{2}(m)} \leqslant \sqrt{\frac{N U \ln ^{3} U}{t}} \leqslant \frac{N \ln ^{3 / 2+\alpha} N}{\sqrt{t}} \leqslant \frac{N}{\ln ^{A} N} .
$$

Следовательно, $\omega\left(n_{2}\right)=\Omega\left(n_{2}\right)$.

Учитывая предыдущие рассуждения, представив $\tau(|b n-a|)$ как сумму по делителям и просуммировав под гиперболой, получим, что сумма (1) равна

$$
2 \sum_{\substack{d \leqslant Q \\(d, b)=1}} \sum_{n_{1} \leqslant y} \sum_{\substack{n_{2} \leqslant N / n_{1} \\ n_{1} n_{2} b \equiv a(\bmod d) \\ \omega\left(n_{1} n_{2}\right)=k}} 1+R_{2}+R_{1}
$$

Здесь и далее считаем, что $Q \leqslant \sqrt{U} / \ln ^{A} N$. Вьпишем и оценим остатки $R_{2}$ и $R_{1}$ в $(5)$. Начнем с $R_{1}$ :

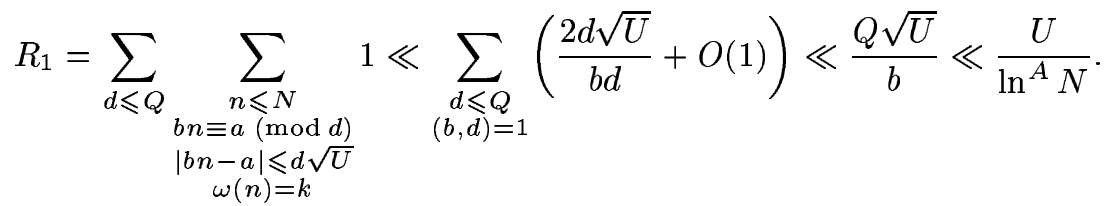

Здесь мы воспользовались тривиальными оценками и тем, что при $|b n-a| \leqslant d \sqrt{U}$

$$
n \in\left[\frac{a-d \sqrt{U}}{b}, \frac{a+d \sqrt{U}}{b}\right] .
$$


Теперь займемся оценкой $R_{2}$. Для этого разобьем его на две суммы следующим образом:

$$
\begin{aligned}
& R_{2}= \sum_{Q \leqslant d \leqslant \sqrt{U}} \sum_{\substack{n \leqslant N \\
b n \equiv a(\bmod d) \\
\omega(n)=k}} 1=\sum_{\substack{\delta \mid a \\
\delta>V}} \sum_{\substack{Q / \delta \leqslant d \leqslant \sqrt{U} / \delta \\
(d, a b / \delta)=1}} \sum_{\substack{n \leqslant N / \delta \\
b n \equiv / \delta(\bmod d) \\
\omega(n \delta)=k}} 1 \\
&+\sum_{\substack{\delta \mid a \\
\delta \leqslant V}} \sum_{\substack{Q / \delta \leqslant d \leqslant \sqrt{U} / \delta \\
(d, a b / \delta)=1}} 1 . \\
& \substack{n \leqslant N / \delta \\
b n=a / \delta(\bmod d) \\
\omega(n \delta)=k}
\end{aligned}
$$

Первая сумма в последнем выражении

$$
\ll \sum_{\substack{\delta \mid a \\ \delta>V}} \sum_{\substack{Q / \delta \leqslant d \leqslant \sqrt{U} / \delta \\(\delta, a b / \delta)=1}} \frac{U}{\delta d} \ll U \ln N \sum_{\substack{\delta \mid a \\ \delta>V}} \frac{1}{\delta} \ll \frac{U \ln N}{V} \tau(a)
$$

а это в силу оценки $\tau(a) \ll a^{\varepsilon}, \varepsilon>0$ (см. [18]) при $V=U^{3 \varepsilon_{1}}, \varepsilon_{1}>0$ есть $O\left((U \ln N) / U^{2 \varepsilon}\right)$. Далее применяем для оценки суммы по $\delta \leqslant V$, входящей в $R_{2}$, лемму 3 , учитывая, что $k \leqslant \gamma \ln _{2} N$. Используя лемму 4 , получим

$$
\begin{aligned}
R_{2} & \ll \sum_{\substack{\delta \mid a \\
\delta \leqslant V}} \sum_{\substack{Q / \delta<d \leqslant \sqrt{U} / \delta \\
(d, a b / \delta)=1}} \frac{N}{\delta \varphi(d) \ln N} \frac{\left(\ln _{2} N\right)^{k-\omega(\delta)}}{(k-\omega(\delta)) !} \sqrt{k}+U^{1-2 \varepsilon} \ln N \\
& \ll \frac{N}{\ln N} \frac{\left(\ln _{2} N\right)^{k-1}}{(k-1) !} \ln \frac{\sqrt{U}}{Q} \sum_{\delta \mid a} \frac{(k-1) ! \delta^{-1}}{(k-\omega(\delta)) !} \frac{1}{\left(\ln _{2} N\right)^{\omega(\delta)-1}} \\
& \ll \frac{N}{\ln N} \frac{\left(\ln _{2} N\right)^{k-1}}{(k-1) !}\left(\ln _{2} N\right)^{2} \ln \frac{\sqrt{U}}{Q} \sum_{\delta \mid a} \frac{\gamma^{\omega(\delta)}}{\delta} \ll \frac{N}{\ln N} \frac{\left(\ln _{2} N\right)^{k-1}}{(k-1) !}(\ln N)^{\varepsilon} .
\end{aligned}
$$

Мы воспользовались тем, что $\ln (\sqrt{U} / Q) \ll(\ln N)^{\varepsilon}$. Представим $\delta \mid a b$ в виде $\delta_{1} \delta_{2}$, где $\delta_{1}$ - делитель $n_{1}$, а $\delta_{2}$ - делитель $n_{2}$. Тогда сумма (1) равна

$$
2 \sum_{\substack{\delta_{1} \delta_{2} \mid a \\ \delta_{1} \delta_{2} \leqslant V}} \sum_{\substack{d \leqslant Q /\left(\delta_{1} \delta_{2}\right) \\\left(d, a b /\left(\delta_{1} \delta_{2}\right)\right)=1}} \sum_{\substack{n_{1} \leqslant y \\\left(n_{1}, d\right)=1}} 1+O\left(\frac{N}{\ln N} \frac{\left(\ln _{2} N\right)^{k-1}}{(k-1) !}(\ln N)^{\varepsilon}\right) .
$$

Здесь мы воспользовались тем, что $n_{2}$ не делится на $p^{2}$ и $\omega\left(n_{2}\right)=\Omega\left(n_{2}\right)$.

Главньй член выражения (6), записанный в удобном для применения леммы 2 виде, после применения этой леммы становится равньм

$$
2 \sum_{\substack{\delta_{1} \delta_{2} \mid a \\ \delta_{1} \delta_{2} \leqslant V}} \sum_{\substack{d \leqslant Q /\left(\delta_{1} \delta_{2}\right) \\\left(d, a b /\left(\delta_{1} \delta_{2}\right)\right)=1}} \frac{1}{\substack{n_{1} \leqslant y \\\left(n_{1}, d\right)=1}} \sum_{\substack{n \leqslant N / \delta \\ \Omega\left(n_{2}\right)=k-\omega\left(n_{1} \delta\right) \\\left(n_{2}, d\right)=1}} 1+R_{3}
$$


где

$$
\begin{aligned}
R_{3}= & \sum_{\substack{\delta \mid a \\
\delta \leqslant V}} \sum_{n_{1} \leqslant y} \sum_{\begin{array}{c}
d \leqslant Q / \delta \\
\left(d, a b n_{1} / \delta\right)=1
\end{array}} 1\left(\mu\left(\frac{N}{\delta n_{1}}, k-\omega\left(n_{1} \delta\right), t, \frac{a}{\delta_{1} \delta_{2}} \bar{n}_{1} \bar{b}, d\right)\right. \\
& \left.-\frac{1}{\varphi(d)}\left|\left\{n_{2}: n_{2} \leqslant \frac{N}{\delta}, \Omega\left(n_{2}\right)=k-\omega\left(n_{1} \delta\right),\left(n_{2}, d\right)=1,\left(n_{2}, P(t)\right)=1\right\}\right|\right) \\
\ll & \sum_{\delta \mid a} \sum_{n_{1} \leqslant y}\left(\frac{Q}{\delta} \sqrt{\frac{N}{\delta n_{1}}} \exp \left(\left(\ln _{2} N\right)^{2+\varepsilon}+\frac{N}{n_{1} \delta}(\ln N)^{-B}\right)\right) \\
\ll & Q \sqrt{N} \exp \left(\left(\ln _{2} N\right)^{2+\varepsilon}\right) \sqrt{y}+\frac{N \ln ^{2} N}{\ln ^{B} N} \ll \frac{N}{\ln ^{A} N}
\end{aligned}
$$

при $Q=\sqrt{N / y} \exp \left(-\left(\ln _{2} N\right)^{5}\right)$ с любой положительной постоянной $A$.

В главном члене выражения (6) продлим интервал изменения $d$ от $Q / \delta$ до $\sqrt{N} / \delta$ (так как часть главного члена при $d \in[Q / \delta, \sqrt{N} / \delta]$ оценивается так же, как и остаток $\left.R_{2}\right)$, поменяем местами суммы по $n_{2}$ и $d$, а затем применим лемму 5 . Тогда главный член примет вид

$$
\begin{aligned}
& 2 \sum_{\substack{\delta \mid a \\
\delta \leqslant V}} \sum_{\substack { n_{1} \leqslant y \\
\begin{subarray}{c}{n_{2} \leqslant N / \delta \\
\Omega\left(n_{2}\right)=k-\omega\left(n_{1} \delta\right){ n _ { 1 } \leqslant y \\
\begin{subarray} { c } { n _ { 2 } \leqslant N / \delta \\
\Omega ( n _ { 2 } ) = k - \omega ( n _ { 1 } \delta ) } }\end{subarray}}\left(\prod_{p}\left(1+\frac{1}{p(p+1)}\right) \prod_{\substack{p \mid \frac{a b n}{\delta}}}\left(1-\frac{1}{p}\right)\left(1+\frac{1}{p(p-1)}\right)^{-1} \ln \frac{\sqrt{N}}{\delta}\right. \\
& \left.+O\left(\sum_{d \mid \frac{a b n}{\delta}} \frac{1+\ln d}{d}\right)\right)
\end{aligned}
$$

Остатки, возникающие при использовании леммы 5 с заменой $\ln (\sqrt{N} / \delta)$ на $\ln \sqrt{N}$, оцениваются следующим образом:

$$
\ll \sum_{\substack{\delta \mid a \\ \delta \leqslant V}} \ln \delta \sum_{\substack{n \leqslant N / \delta \\ \Omega\left(n_{2}\right)=k-\omega\left(n_{1} \delta\right)}} 1+\sum_{\substack{\delta \mid a \\ \delta \leqslant V}} \sum_{\substack{n \leqslant N / \delta \\ \Omega\left(n_{2}\right)=k-\omega\left(n_{1} \delta\right)}} \frac{1+\ln d}{d \mid \frac{a b n}{\delta}}
$$

Учитьвая, что $k \leqslant \gamma \ln _{2} N, \omega\left(n_{1} \delta\right) \leqslant \omega\left(n_{1}\right)+\omega(\delta)$, используя лемму 4 и лемму 1 при $t=\exp \left(\left(\ln _{2} N\right)^{2}\right)$, получим, что последнее выражение

$$
\begin{aligned}
& \ll \ln _{2}^{2} N \sum_{\substack{\delta \mid a \\
\delta \leqslant V}}(1+\ln \delta) \sum_{n_{1} \leqslant y} \sum_{\substack{n_{2} \leqslant N /\left(\delta n_{1}\right) \\
\omega\left(n_{2}\right)=k-\omega\left(n_{1} \delta\right)}} 1 \\
& \ll \ln _{2}^{2} N \frac{N}{\ln N} \frac{\left(\ln _{2} N\right)^{k-1}}{(k-1) !} \sum_{\delta \mid a} \frac{\gamma^{\omega(\delta)}(1+\ln \delta)}{\delta} \sum_{\substack{n_{1} \leqslant y \\
\omega\left(n_{1} \delta\right) \leqslant k}} \gamma^{\omega\left(n_{1}\right)} n_{1}^{-1} \\
& \ll \frac{N}{\ln N(k-1) !} \ln _{2}^{k-1} N \ln ^{\varepsilon} N .
\end{aligned}
$$

Итак, в обозначениях теоремы 3 и леммы 1 задача свелась к исследованию суммы

$$
\sum_{\substack{\delta \mid a \\ \delta \leqslant V}} \sum_{\substack{n_{1} \leqslant y \\ \omega\left(n_{1} \delta\right) \leqslant k}} \prod_{\substack{a \mid \frac{a b n_{1}}{\delta} \\ \delta}} \lambda(p) \boldsymbol{C} \ln N \pi\left(\frac{N}{n_{1} \delta}, t, k-\omega\left(n_{1} \delta\right)\right)\left(1+O\left(\frac{1}{\ln ^{2} N}\right)\right) .
$$


Мы воспользовались тем, что произведение $\lambda(p)$ можно производить только по $p \leqslant t$. Действительно, если $n_{2}=A \leqslant N^{\alpha}$, то

$$
\prod_{\substack{p>t \\ p \mid A}} \lambda(p)=\exp \left(O\left(\sum_{\substack{p>t \\ p \mid A}} \frac{p}{p^{2}-p+1}\right)\right)=\exp \left(O\left(\frac{\ln N}{t}\right)\right)=1+O\left(\frac{1}{\ln ^{2} N}\right) .
$$

Прежде чем воспользоваться леммой 1, сделаем некоторые преобразования. Заметим, что $\ln t \leqslant(\ln N)^{\varepsilon}, \delta n_{1} \leqslant N^{\varepsilon} ;$ поэтому

$$
z=\frac{\ln \left(N /\left(\delta n_{1}\right)\right)}{\ln t} \geqslant(1-\varepsilon)(\ln N)^{1-\varepsilon}, \quad \ln z \geqslant \frac{1}{2} \ln _{2} N .
$$

Следовательно, учитьвая, что $k \leqslant \gamma \ln _{2} N$ и $r=\left(k-1-\omega\left(n_{1} \delta\right)\right) / \ln z$, имеем

$$
\prod_{p>t}\left(1-\frac{1}{p}\right)^{r}\left(1+\frac{r}{p-1}\right)=\exp \left(r \sum_{p>t}\left(\frac{1}{p}-\frac{1}{p-1}\right)+O\left(\frac{1}{t}\right)\right)=1+O\left(\frac{1}{\ln N}\right) .
$$

Пусть $\sigma=(k-1) / \ln _{2} N$. Тогда

$$
|r-\sigma| \ll(k-1) \frac{\left|\ln z-\ln _{2} N\right|}{\left(\ln _{2} N\right)^{2}}+\frac{\omega\left(n_{1} \delta\right)}{\ln _{2} N} .
$$

Отсюда, учитывая, что

$$
\ln z-\ln _{2} N=\ln \frac{z}{\ln N}=\ln \left(1-\frac{\ln \delta n_{1}}{\ln N}\right)-\ln _{2} t
$$

получаем

$|r-\sigma| \ll(k-1) \frac{\ln \delta n_{1}}{\ln N\left(\ln _{2} N\right)^{2}}+(k-1) \frac{\ln _{2} t}{\left(\ln _{2} N\right)^{2}}+\frac{\omega\left(n_{1} \delta\right)}{\ln _{2} N} \ll \frac{\ln \delta n_{1}}{\ln N \ln _{2} N}+\frac{\ln _{2} t}{\ln _{2} N}+\frac{\omega\left(n_{1} \delta\right)}{\ln _{2} N}$.

Поэтому

$$
\begin{aligned}
& \frac{1}{\Gamma(r+1)}-\frac{1}{\Gamma(\sigma+1)}=\left(\frac{1}{\Gamma(x)}\right)_{\theta}^{\prime}(r-\sigma) \ll \frac{1}{\Gamma(\sigma+1)}|r-\sigma| \\
& \quad \ll \frac{1}{\Gamma(\sigma+1)}\left(1+O\left(\frac{\ln \delta n_{1}}{\ln N}+\frac{\ln _{2} t}{\ln _{2} N}+\frac{\omega\left(n_{1} \delta\right)}{\ln _{2} N}\right)\right), \quad \theta \in(r+1, \sigma+1) .
\end{aligned}
$$

Положим $T=k-\omega\left(n_{1} \delta\right)-1$. Имеем

$$
\begin{aligned}
(\ln z)^{T}(\ln t)^{T / \ln z}= & \left(\ln _{2} \frac{N}{\delta n_{1}}\right)^{T}\left(1-\frac{\ln _{2} t}{\ln _{2} \frac{N}{\delta n_{1}}}\right)^{T}(\ln t)^{T\left(\ln _{2} \frac{N}{\delta n_{1}}\right)^{-1}\left(1-\ln _{2} t / \ln _{2} \frac{N}{\delta n_{1}}\right)^{-1}} \\
= & \left(\ln _{2} N\right)^{T}\left(1+O\left(\frac{\ln \delta n_{1}}{\ln N \ln _{2} N}\right)\right)^{T} \exp \left(T \ln \left(1-\frac{\ln _{2} t}{\ln _{2} \frac{N}{\delta n_{1}}}\right)\right. \\
& \left.+T \ln _{2} t\left(1-\frac{\ln _{2} t}{\ln _{2} \frac{N}{\delta n_{1}}}\right)^{-1}\left(\ln _{2} \frac{N}{\delta n_{1}}\right)^{-1}\right) \\
= & \left(\ln _{2} N\right)^{T}\left(1+O\left(\frac{\ln \delta n_{1}}{\ln N}+\frac{\ln _{2}^{2} t}{\ln _{2} N}\right)\right)
\end{aligned}
$$


Учитывая последнее соотношение, получим

$$
\begin{aligned}
\pi\left(\frac{N}{\delta n_{1}}, t, k-\omega\left(\delta n_{1}\right)\right)= & \frac{1}{\Gamma(\sigma+1)} \frac{N}{\delta n_{1} \ln N} \frac{\left(\ln _{2} N\right)^{k-1-\omega\left(\delta n_{1}\right)}}{\left(k-1-\omega\left(\delta n_{1}\right)\right) !} \\
& \times \prod_{p \leqslant t}\left(1-\frac{1}{p}\right)^{r}\left(1+O\left(\frac{\ln n_{1} \delta}{\ln N}+\frac{\ln _{2}^{2} t}{\ln _{2} N}+\frac{\omega\left(n_{1} \delta\right)}{\ln _{2} N}\right)\right) .
\end{aligned}
$$

Прежде чем подставить полученньй результат в (7), заметим, что

$$
\begin{aligned}
\frac{\left(\ln _{2} N\right)^{k-1-\omega\left(n_{1} \delta\right)}}{\left(k-1-\omega\left(n_{1} \delta\right)\right) !} & =\frac{\left(\ln _{2} N\right)^{k-1}}{(k-1) !} \frac{(k-1) \cdots\left(k-\omega\left(n_{1} \delta\right)\right)}{(\ln N) \omega\left(\delta n_{1}\right)} \\
& =\frac{\left(\ln _{2} N\right)^{k-1}}{(k-1) !}\left(1+O\left(\frac{\omega^{2}\left(n_{1} \delta\right)}{k}\right)\right)\left(\frac{k}{\ln _{2} N}\right)^{\omega\left(\delta n_{1}\right)} .
\end{aligned}
$$

Кроме того, если обозначить $\sigma_{1}=\sigma-\omega\left(n_{1} \delta\right)\left(\ln _{2} N\right)^{-1}$, то

$$
\prod_{p \leqslant t}\left(1-\frac{1}{p}\right)^{r}=\prod_{p \leqslant t}\left(1-\frac{1}{p}\right)^{\sigma_{1}} \exp \left(O\left(\left|r-\sigma_{1}\right| \ln _{2} t\right)\right)
$$

Таким образом, учитьвая (8), имеем

$$
\prod_{p \leqslant t}\left(1-\frac{1}{p}\right)^{r}=\prod_{p \leqslant t}\left(1-\frac{1}{p}\right)^{\sigma} \prod_{p \leqslant t}\left(1-\frac{1}{p}\right)^{-\omega\left(n_{1} \delta\right) / \ln _{2} N}\left(1+O\left(\frac{\ln \delta n_{1}}{\ln N}+\frac{\ln _{2} t}{\ln _{2} N}\right)\right) .
$$

Подставим полученньй результат в (7), отбрасьвая условие $p \leqslant t$ в произведении $\lambda(p)$ и, кроме того, условия $\delta \leqslant V, n_{1} \leqslant y$ (можно отбросить, ведь остатки оцениваются как и ранее), $\omega\left(n_{1} \delta\right) \leqslant k$ (можно отбросить, так как среди остатков есть $\omega^{2}\left(n_{1} \delta\right) k^{-1} \geqslant 1$ при $\left.\omega\left(n_{1} \delta\right) \geqslant k\right)$. Получим, что (7) равно

$$
\begin{aligned}
& \frac{C N}{\Gamma(\sigma+1)} \frac{\left(\ln _{2} N\right)^{k-1}}{(k-1) !} \prod_{p \leqslant t}\left(1-\frac{1}{p}\right)^{\sigma}\left(1+O\left(\frac{\left(\ln _{2} t\right)^{2}}{\ln _{2} N}+\frac{\ln y}{\ln N}\right)\right) \\
& \times \sum_{\delta \mid a} \frac{1}{\delta} \sum_{n_{1}} \frac{1}{n_{1}} \prod_{p \mid \frac{a b n_{1}}{\delta}} \lambda(p) \mu^{\omega\left(n_{1} \delta\right)}\left(1+O\left(\frac{\ln \delta}{\ln N}+\frac{\omega^{2}\left(n_{1} \delta\right)}{k}\right)\right),
\end{aligned}
$$

где

$$
\mu=\frac{k}{\ln _{2} N} \prod_{p \leqslant t}\left(1-\frac{1}{p}\right)^{-1 / \ln _{2} N}=\frac{k}{\ln _{2} N}\left(1+O\left(\frac{\ln _{2} t}{\ln _{2} N}\right)\right) .
$$

Оценим остаток, возникающий за счет $O\left(\ln \delta(\ln N)^{-1}\right)$. Так как $\lambda(p) \geqslant 1 / 3$ и $\mu \leqslant 2 \gamma$, имеем

$$
\begin{aligned}
\sum_{\delta \mid a} & \frac{1}{\delta} \sum_{n_{1}} \frac{1}{n_{1}} \prod_{p \mid \frac{a b n_{1}}{\delta}} \lambda(p) \mu^{\omega\left(n_{1} \delta\right)} \frac{\ln \delta}{\ln N} \\
& \leqslant \frac{1}{\ln N} \sum_{q^{r} \delta \mid a} \frac{\ln q^{r}}{\delta q^{r}} \sum_{n_{1}} \frac{1}{n_{1}} \cdot 3 \prod_{p \mid \frac{a b n_{1}}{\delta}} 2 \gamma \lambda(p) \mu^{\omega\left(n_{1} \delta\right)} \\
& \leqslant \frac{6 \gamma}{\ln N} \sum_{q^{r} \mid a} \frac{\ln q^{r}}{q^{r}} \sum_{\delta \mid a} \frac{1}{\delta} \sum_{n_{1}} \frac{1}{n_{1}} \prod_{p \mid \frac{a b n_{1}}{\delta}} \lambda(p) \mu^{\omega\left(n_{1} \delta\right)}
\end{aligned}
$$


Применим лемму 4 и отбросим этот остаток, так как он

$$
\begin{aligned}
& \ll \frac{C N}{\Gamma(\sigma+1)} \frac{\left(\ln _{2} N\right)^{k-1}}{(k-1) !} \frac{\ln _{2}^{2} N}{\ln N} \sum_{\delta \mid a} \frac{1}{\delta} \sum_{n_{1}} \frac{1}{n_{1}} \prod_{p \mid \frac{a b n_{1}}{\delta}} \lambda(p) \mu^{\omega\left(n_{1} \delta\right)} \\
& \ll \frac{C N}{\ln N} \frac{\left(\ln _{2} N\right)^{k-1}}{(k-1) !}(\ln N)^{\varepsilon} .
\end{aligned}
$$

Остается оценить остаток с $\omega^{2}\left(n_{1} \delta\right) / k$. Рассмотрим функцию

$$
\Phi(v)=\sum_{\delta \mid a} \sum_{n_{1}} \frac{1}{\delta n_{1}} \prod_{\delta p \mid a b n_{1}} \lambda(p) v^{\omega\left(n_{1} \delta\right)} .
$$

Преобразуем ее:

$$
\Phi(v)=\sum_{\delta \mid a} \prod_{p \mid \frac{a b}{\delta}} \lambda(p) v^{\omega(\delta)} \sum_{n_{1}} \frac{v^{\omega\left(n_{1} \delta\right)-\omega(\delta)}}{n_{1}} \prod_{\substack{q \mid \frac{a b n_{1}}{\delta} \\ q \nmid \frac{a b}{\delta}}} \lambda(q) .
$$

Сумма по $n_{1}$ равна

$$
\begin{aligned}
& \prod_{\substack{p \nmid a b \\
p \leqslant t}}\left(1+\frac{\lambda(p) v}{p-1}\right) \prod_{p \mid b}\left(1+\frac{v}{p-1}\right) \prod_{\substack{p \mid \delta \\
p \nmid \frac{a}{\delta}}}\left(1+\frac{\lambda(p)}{p-1}\right) \\
& \times \prod_{p \mid \delta}\left(1+\frac{\lambda(p)}{p-1}\right) \prod_{\substack{p|\delta \\
p| \frac{a}{\delta}}}\left(1-\frac{1}{p}\right)^{-1}\left(1+\frac{\lambda(p)}{p-1}\right)^{-1}\left(1+\frac{v}{p-1}\right)^{-1} \prod_{p \mid \frac{a}{\delta}}\left(1+\frac{v}{p-1}\right) \\
& =\prod_{\substack{p \nmid a b \\
p \leqslant t}}\left(1+\frac{\lambda(p) v}{p-1}\right) \prod_{p \mid a}\left(1+\frac{v}{p-1}\right) \prod_{p \mid b}\left(1+\frac{v}{p-1}\right) \\
& \times \prod_{p \mid \delta}\left(1+\frac{\lambda(p)}{p-1}\right)\left(1+\frac{v}{p-1}\right)^{-1} \prod_{\substack{p|\delta \\
p| \frac{a}{\delta}}}\left(1-\frac{1}{p}\right)^{-1}\left(1+\frac{\lambda(p)}{p-1}\right)^{-1}\left(1+\frac{v}{p-1}\right)^{-1} .
\end{aligned}
$$

Таким образом,

$$
\Phi(v)=\prod_{\substack{p \nmid a b \\ p \leqslant t}}\left(1+\frac{\lambda(p) v}{p-1}\right) \prod_{p \mid a b}\left(1+\frac{v}{p-1}\right) \lambda(p) \Psi(v),
$$

где

$$
\begin{aligned}
\Psi(v)= & \sum_{\delta \mid a} \frac{1}{\delta} v^{\omega(\delta)} \prod_{p \mid \delta}\left(1+\frac{\lambda(p)}{p-1}\right) \frac{1}{\lambda(p)} \prod_{\substack{p|\delta \\
p| \frac{a}{\delta}}}\left(1-\frac{1}{p}\right)^{-1}\left(1+\frac{\lambda(p)}{p-1}\right)^{-1}\left(1+\frac{v}{p-1}\right)^{-1} \\
= & \prod_{p \mid a}\left(1+\frac{v}{\lambda(p)}\left(1-\frac{1}{p}\right)^{-2}\left(1+\frac{v}{p-1}\right)^{-1}\left(\frac{1}{p}-\frac{1}{p^{\alpha(a, p)}}\right)\right. \\
& \left.+\frac{v}{p^{\alpha(a, p)}}\left(1+\frac{\lambda(p)}{p-1}\right) \frac{1}{\lambda(p)}\right) .
\end{aligned}
$$


Напомним, что $\alpha(a, p)$ - показатель степени, с которой $p$ входит в каноническое разложение $a$. Далее,

$$
\begin{aligned}
\Phi(v)= & \prod_{\substack{p \nmid a b \\
p \leqslant t}}\left(1+\frac{\lambda(p) v}{p-1}\right) \prod_{p \mid a}\left(\lambda(p)+\frac{\lambda(p) v}{p-1}+v\left(1-\frac{1}{p}\right)^{-2}\left(\frac{1}{p}-\frac{1}{p^{\alpha(a, p)}}\right)\right. \\
& \left.+\frac{v}{p^{\alpha(a, p)}}\left(1+\frac{\lambda(p)}{p-1}\right)\left(1+\frac{v}{p-1}\right)\right) \prod_{p \mid b}\left(1+\frac{v}{p-1}\right) \lambda(p) .
\end{aligned}
$$

Теперь запишем $\Phi(v)$ в следующем виде:

$$
\Phi(v)=\prod_{p \leqslant t} f(p, a, b, v) \prod_{p \mid a b} g(p, a, b, v)
$$

причем при $0 \leqslant v \leqslant 2 \gamma$ функции $f(p, a, b, v)=f, g(p, a, b, v)=g$ обладают следующими свойствами: $1 \leqslant f \leqslant c, 1 / 3 \leqslant g \leqslant c,\left|f^{\prime}\right|+\left|f^{\prime \prime}\right| \ll 1 / p,\left|g^{\prime}\right|+\left|g^{\prime \prime}\right| \ll 1 / p, c$ - постоянная. Поэтому имеем

$$
\begin{aligned}
\frac{v}{k}\left(v \Phi^{\prime}(v)\right)^{\prime}= & \frac{1}{k}\left(v \Phi^{\prime}(v)+v^{2} \Phi^{\prime \prime}(v)\right) \\
= & \frac{1}{k}\left(v\left(\sum_{p \leqslant t} \frac{f^{\prime}}{f}+\sum_{p \mid a b} \frac{g^{\prime}}{g}\right) \Phi(v)+v^{2}\left(\sum_{p \leqslant t} \frac{f^{\prime}}{f}+\sum_{p \mid a b} \frac{g^{\prime}}{g}\right)^{2} \Phi(v)\right. \\
& \left.+v^{2}\left(\sum_{p \leqslant t} \frac{f^{\prime \prime} f-\left(f^{\prime}\right)^{2}}{f}+\sum_{p \mid a b} \frac{g^{\prime \prime} g-\left(g^{\prime}\right)^{2}}{g}\right) \Phi(v)\right)
\end{aligned}
$$

Отсюда находим

$$
\begin{aligned}
\sum_{\delta \mid a} \frac{1}{\delta} \sum_{n_{1}} \frac{1}{n_{1}} \prod_{p \mid \frac{a b n_{1}}{\delta}} \lambda(p) \mu^{\omega\left(n_{1} \delta\right)} \frac{\omega^{2}\left(n_{1} \delta\right)}{k} & =\left.\frac{v}{k}\left(v \Phi^{\prime}(v)\right)^{\prime}\right|_{v=\mu} \\
& \ll \frac{1}{k} \mu \Phi(\mu)\left(\ln _{2} t+\sum_{p \mid a b} \frac{1}{p}\right) \ll \frac{\ln _{2} t}{\ln _{2} N} \Phi(\mu)
\end{aligned}
$$

Мы воспользовались здесь равенством $(9)$ и оценкой $\sum_{p \mid a b}(1 / p) \ll \ln _{3} N$. Следовательно, главньй член имеет вид

$$
\frac{C N}{\Gamma(\sigma+1)} \frac{\left(\ln _{2} N\right)^{k-1}}{(k-1) !} \prod_{p \leqslant t}\left(1-\frac{1}{p}\right)^{(k-1) / \ln _{2} N} \Phi(\mu)\left(1+O\left(\frac{\left(\ln _{2} t\right)^{2}}{\ln _{2} N}\right)\right)
$$

Наша задача - избавиться с нужной точностью от зависимости от $t$ произведения

$$
\prod_{p \leqslant t}\left(1-\frac{1}{p}\right)^{(k-1) / \ln _{2} N} \Phi(\mu)
$$


Пусть

$$
\mu_{0}=\frac{k-1}{\ln _{2} N} \prod_{p \leqslant t}\left(1-\frac{1}{p}\right)^{-1 / \ln _{2} N}=\sigma \prod_{p \leqslant t}\left(1-\frac{1}{p}\right)^{-1 / \ln _{2} N} .
$$

Воспользуемся первьм равенством из (9); получим

$$
\Phi(\mu)-\Phi\left(\mu_{0}\right)=\Phi^{\prime}(\theta)\left(\mu-\mu_{0}\right)=\Phi(\theta) O\left(\frac{\Phi^{\prime}(\theta)}{\Phi(\theta)}\left|\mu-\mu_{0}\right|\right) \ll \Phi(\theta) \frac{\ln _{2} t}{\ln _{2} N},
$$

где $\mu_{0}<\theta<\mu$ и $\Phi(\theta) \leqslant \Phi(\mu)$, т.е.

$$
\Phi(\mu)=\Phi\left(\mu_{0}\right)\left(1+O\left(\frac{\ln _{2} t}{\ln _{2} N}\right)\right) .
$$

Обозначим

$$
E_{a, b}(v)=\prod_{p \mid a b} g(p, a, b, v)
$$

Тогда

$$
E_{a, b}^{\prime}(v) \ll \sum_{p \mid a b} \frac{1}{p} \cdot E_{a, b}(v) \ll \ln _{3} N E_{a, b}(v) .
$$

Следовательно, учитьвая, что $\left|\sigma-\mu_{0}\right| \ll \ln _{2} t\left(\ln _{2} N\right)^{-1}$, имеем

$$
E_{a, b}\left(\mu_{0}\right)=E_{a, b}(\sigma)\left(1+O\left(\frac{\ln _{3} N \ln _{2} t}{\ln _{2} N}\right)\right) .
$$

Рассмотрим произведение при $\sigma=(k-1) / \ln _{2} N$ :

$$
\begin{aligned}
\prod_{p \leqslant t} & \left(1-\frac{1}{p}\right)^{\sigma}\left(1+\sigma \prod_{q \leqslant t}\left(1-\frac{1}{q}\right)^{-1 / \ln _{2} N} \frac{\lambda(p)}{p-1}\right) \\
& =\prod_{p \leqslant t}\left(1-\frac{1}{p}\right)^{\sigma}\left(1+\sigma \frac{\lambda(p)}{p-1}\right)\left(1+O\left(\frac{\ln _{2} t}{\ln _{2} N}\right)\right) \\
& =\prod_{p}\left(1-\frac{1}{p}\right)^{\sigma}\left(1+\sigma \frac{\lambda(p)}{p-1}\right)\left(1+O\left(\frac{\ln _{2} t}{\ln _{2} N}\right)\right) .
\end{aligned}
$$

Окончательно, учитьвая, что $t=\exp \left(\left(\ln _{2} N\right)^{2}\right)$, находим, что главный член (1) равен

$$
\frac{C N}{\Gamma(\sigma+1)} \frac{\left(\ln _{2} N\right)^{k-1}}{(k-1) !} \prod_{p}\left(1-\frac{1}{p}\right)^{\sigma}\left(1+\sigma \frac{\lambda(p)}{p-1}\right) E(a, b, \sigma),
$$

где

$$
\begin{aligned}
E(a, b, \sigma)= & \prod_{p \mid a}\left(\lambda(p)+\frac{\sigma \lambda(p)}{p-1}+\sigma\left(1-\frac{1}{p}\right)^{-2}\left(\frac{1}{p}-\frac{1}{p^{\alpha(a, p)}}\left(1+\frac{\lambda(p)}{p-1}\right)\left(1+\frac{\sigma}{p-1}\right)\right)\right) \\
& \times \prod_{p \mid b}\left(1+\frac{\sigma}{p-1}\right) \lambda(p) \prod_{p \mid a b}\left(1+\sigma \frac{\lambda(p)}{p-1}\right)^{-1}\left(1+O\left(\frac{\left(\ln _{3} N\right)^{2}}{\ln _{2} N}\right)\right) .
\end{aligned}
$$


Это выражение действительно можно считать главным членом, так как

$$
\begin{aligned}
E(a, b, \sigma) & \geqslant \prod_{p \mid a b}\left(1+\sigma \frac{\lambda(p)}{p-1}\right)^{-1} \prod_{p \mid a}\left(1-\frac{p}{p^{2}-p+1}\right) \\
& \geqslant c+\exp \left(-\sigma \sum_{p \mid a b} \frac{1}{p}\right) \geqslant c_{1}\left(\ln _{2} N\right)^{-\sigma-c_{2}}>c_{1}(\ln N)^{-\varepsilon},
\end{aligned}
$$

где $c_{1}>0$.

Учитывая оценки остатков, полученные вьше, приходим к утверждению теоремы 3.

В заключение автор выражает искреннюю благодарность профессору Н. М. Тимофееву за полезные советы по содержанию работы.

\section{СПИСОК ЦИТИРОВАННОЙ ЛИТЕРАТУРЫ}

[1] Titchmarsh E. C. A divisor problem // Rend. Circ. Mat. Palermo. 1930. V. 54. P. 414-429.

[2] Линник Ю.В. Дисперсионный метод в бинарных аддитивных задачах. Л.: Изд-во ЛГУ, 1961.

[3] Бредихин Б. М. Бинарные аддитивные проблемы неопределенного типа. I // Изв. АН СССР. Сер. матем. 1963. Т. 27. № 2. С. 439-462.

[4] Барбан М. Б. Об аналогах проблемы делителей Титчмарша // Вестн. ЛГУ. Матем., мех., астрон. 1963. Т. 19. С. 5-13.

[5] Каршнев А.К. Обобшение проблемы делителей Титчмарша // Изв. АН УзССР. Сер. физ.-мат. наук. 1969. Т. 13. №1. С. 69-70.

[6] Пиядина ЖК.В. Аддитивные задачи с почти простыми числами // Всесоюзная школа "Конструктивные методы и алгоритмы теории чисел". Тезисы докл. Минск, 1989.

[7] Fujii A. On some analogues of Titchmarsh divisor problem // Nagoya Math. J. 1976. V. 64. P. $149-158$.

[8] Тимофеев Н. М., Хрипунова М. Б. Распределение чисел с заданньм числом простых делителей в прогрессиях // Матем. заметки. 1994. Т. 55. № 2. С. 144-156.

[9] Тимофеев Н. М., Хрипунова М. Б. Проблема Титчмарша с числами, имеющими заданное число простых делителей // Матем. заметки. 1996. Т. 59. № 4. С. 586-603.

[10] Selberg A. Note on a paper by L. G. Sathe // J. Indian Math. Soc. (N.S.). 1954. V. 18. P. 83-87.

[11] Sathe L. G. On a problem of Hardy on the distribution of integers having a given member of prime factors // J. Indian Math. Soc. (N.S.). 1953. V. 17. P. 63-141; 1954. V. 18. P. 27-81.

[12] Nicolas J.-L. Sur la distribution des nombres entiers ayant une quantité fixée de facteurs premiers // Acta Arith. 1984. V. 44. P. 191-200.

[13] Hildebrand A., Tenenbaum G. On the number of prime factors of an integer // Duke Math. J. 1988. V. 56. P. 471-501.

[14] Balazard M. Unimodalité de la distribution du nombre de diviseurs premiers d'un entier // Ann. Inst. Fourier (Grenoble). 1990. V. 40. № 2. P. 255-270.

[15] Alladi K. The distribution of $\nu(n)$ in the sieve of Eratosthenes // Quart. J. Math. Oxford. Ser. (2). 1982. V. 33. P. 129-148.

[16] Shiu P. A Brun-Titchmarsh theorem for multiplicative functions // J. Reine Angew. Math. 1980. V. 313. P. 161-170.

[17] Halberstam H., Richert H.-E. Sieve Methods. London-New York: Acad. Press, 1974.

[18] Виноградов И. М. Метод тригонометрических сумм в теории чисел. М.: Наука, 1971.

[19] Прахар И. М. Распределение простых чисел. М.: Мир, 1967. 